\title{
Die postfoetale Histiogenese des Hodens der Maus bis zur Pubertät.
}

Von

\section{Dr. Friedrich Fermann,}

Docent an dem anatomischen Institut der Tniversität Erlangen.

Hierzu Tafel XXVI.

Der Grund, der mir die Aufnahme von Untersuchungen über diesen Gegenstand wünschenswerth erscheinen liess, bestand nicht sowohl in einer gewissen Neugierde, ein Gebiet, das heutzutage noch als ziemlich unbekannt gelten kann, zu betreten, als vielmehr in etwas ganz anderem. Bekanntlich liegt eine der Hauptschwierigkeiten, welche der Hoden des erwachsenen Sängethieres einer histologischen Analyse entgegenstellt, darin, dass sich in der epithelialen Wand des Samenkanälehens zwei Processe zu gleicher Zeit abspielen, erstens die eigentliche Spermatogenese, die Entstehung der Samenelemente aus ihren zelligen Vorläufern, und dann der Vorgang einer ausgiebigen Regeneration, welche die durch die Spermatogenese in Verlust gerathenen Zellelemente wieder zu ersetzen bestimmt ist. Stellen wir uns diese beiden Vorgänge unter dem Bilde zweier Kreislinien vor, so mlissten wir für die erstere die Vereinigung der Spermatiden mit einer B e nda'schen Fusszelle als Anfangspunkt, das im Lumen des Hodenkanälchens freiliegende Spermatozoon als Schlusspunkt betrachten, während die zweite mit der Neubildung von Spermatogonien beginnen, mit der reifen Spermatide endigen würde. 
Eine neue Schwierigkeit entsteht nun dadurch, dass die Anfangspunkte beider Kreislinien im Epithellager des Samenkanälchens nicht örtlich zusammenfallen, d. h. dass die Neubildung von Spermatogonien nicht mit der ersten Phase der Spermatogenese correspondirt, sondern dass vielmehr die beiden Kreislinien etwas aneinander verschoben sind. Eineu Beweis, dass gerade durch diesen letzteren Umstand so viel des Verwirrenden und Unsicheren in die Histologie des Hodens herein gekommen ist, brauche ich wohl nicht erst zu erbringen, genitgt doch schon ein ziemlich oberflächlicher Blick in die einschlägige Litteratur, nicht nur der früheren, sondern auch der neuesten Zeit, um dies zu bestätigen.

Es dürfte nun zu erwarten sein, dass gerade eine Verfolgung der postfötalen Histiogenese des Hodens bis in die Pubertätszeit binein geeignet sei, in diese Verbältnisse Klarheit zu bringen; können wir doch bei dem jugendlichen Tuiere den einen Kreis, den Vorgang der Spermatogenese, von vorneherein ausschalten, wodurch der andere nur umso deutlicher hervortreten wird, wobei der Voraussetzung Raum gegeben werden diurfte, dass der Process des Wachsthums im jugendlichen, der der Regeneration im erwachsenen, functionirenden Hoden gleiche Bahnen einschlagen wird.

Endlich galt es noch für einen weiteren Punkt einen sicheren Beweis zu liefern. Ich habe mich in einer friiheren Arbeit ${ }^{1}$ ) auf die Seite derjenigen Autoren gestellt, die in den sog. Bendaschen Fusszellen Elemente erblicken, welche bei dem spermatogenetischen Process an und für sich volikommen unbetheiligt sind und nur als Stützelemente fungiren, an denen angelagert die Spermatiden ihre Reifung in Spermatozoen durehmachen; ich habe für diese Ansicht darin einen neuen Beweis erbracht zu haben geglaubt, dass es mir gelang, in dem Kerwe der $B$ e $\mathrm{n} d$ a'scben Fusszelle eine eigenthümliche Nucleolarbildung nachzuweisen, die sich während des ganzen Vorganges der Samenbildung in gleich typischer Weise erbält. Dieser Beweis dïrfte danu noch mehr an Sicherbeit gewinnen, wenn es gelingen wiurde, auch in ganz jugendlichen Hoden schon Kerne anfzufinden, die die characteristische Nucleolarbildung beherbergen, wenn es möglich wäre fest-

1) Beiträge zur Histologie des Hodens. Archiv f. mikr. Anat., Bd. 34, Heft 1. 
Die postfoetale Histiogenese des Hodens der Maus bis zur Pubertät. 431

zustellen, dass von vorneherein bei dem Aufbaue des Hodens zweierlei Elemente betheiligt sind, einerseits Stützzellen, andererseits die eigentlichen Drïsen- oder Samenbildungselemente. In wieweit nun die durch die Untersuchnng zu Tage geförderten thatsächlichen Verhältnisse den eben principiell aufgestellten Gesichtspunkten entsprechen, das soll den Inhalt der folgenden Zeilen bilden.

Vorerst wollen wir uns nun einen Ueberblick verschaffen über die recht spärlichen Angaben, die über unseren Gegenstand in der Litteratur zu finden sind. Der erste, der sich mit der Structur des noch nicht geschlechtsreifen Hodens befasste, ist v. I a Vale tte $\mathrm{S}$ t. G e or g e ${ }^{1}$ ), welcher die Verhältnisse beim Kalbe, Kaninchen, Hunde und beim Menschen untersnchte. Das nicht geschlechtsreife Hodenkanälchen birgt nach diesem Autor, eingebettet in eine das Canallumen vollständig erfüllende Eiweissmasse, zweierlei Kerne, erstens kleine, von runder oder ovaler Form, und zweitens, in unregelmässigen Abständen zwischen jene vertheilt, grössere runde Kerne, die von einer Lage nach aussen scharf abgegrenzten Protoplasmas umgeben sind; diese Gebilde stellen die Spermatogonien dar, die sich innerhalb des Lagers der ersteren Gebilde, der sog. Follikelzellen, vermehren. Es nimmt also v. la V a let te St. G e orge innerhalb der jugendlichen Samenkanälchen zweierlei Elemente an.

Ganz im Gegensatz zu dieser Ansicht v. la Valette St. George's steht Biondic), dem sich im Wesentlichen auch $\mathrm{N}$ iess in $\mathrm{g}^{3}$ ) anschliesst. Für B i on di durfte es ja im Hoden nicht geschleehtsreifer Thiere nur Elemente von einerlei Natur geben, leugnet er doch auch für den functionirenden Hoden das Vorkommen stützender Zellelemente (Follikelzellen, B e n d a'sche Fusszellen) vollkommen. So sehen wir denn auf den Abbildungen, die die Biondi'sche Beschreibung begleiten und die dem Hoden des Kalbes entnommen sind, weiter nichts als runde Zellkerne, welche in eine Zwischensubstanz eingebettet sind, die durch die

1) Ueber die Genese der Samenkörper. Archiv f. mikr. Anat., Bd. 15.

2) Die Entwicklung der Spermatozoiden. Archiv f. mikr. Anat., Bd. 2\%.

3) Untersuchungen über die Entwicklung und den feinsten Bau der Samenfäden einiger Säugethiere. Verhandlungen der phys.-medic. Gesellschaft Würzburg, Bd. XXII, Nr. 2. 
Wirkung härtender Agentien ein eigenthümlich netzartig zerklüftetes Gefüge annimmt. Auch die, nebenbei gesagt, recht mangelhaften Zeichnungen $\mathrm{N}$ i essing's lassen von einer Doppelnatur der das Hodenkanälchen zusammensetzenden Elemente nichts erkennen.

Meine Untersuchungen nun, zu denen junge weisse Mäuse von den ersten Lebensstunden bis in die 6 . Woche hinein verwendet wurden, vermochten, um diesen Punkt gleich von vorneherein zu betonen, die Ansicht v. la Valette St. Ge org e's vollständig $\mathrm{zu}$ bestätigen.

Feine Querschnitte durch die Hoden neugeborener Mäuse lassen nach Anwendung der in einer früheren Arbeit ${ }^{1}$ ) ausführlich angegebenen Härtungs- und Tinctionsmethode zur Evidenz erkennen, dass das Hodenkanälchen zu dieser Zeit, wie dies ja schon von v. la Valette St. Ge orge angegeben wird, noch kein eigentliches Lumen besitzt, vielmehr durchaus von einer Protoplasmamasse ausgefüllt wird. Leuchtende, feine aber deutliche Linien, welche diese Masse durchziehen, Iehren aber, dass wir es nicht mit einer structurlosen Eiweissmasse, einer homogenen Zwischensubstanz zu thun haben, wie dies neben v. I a Valette St. G e or ge auch Biondi behauptet, sondern dass sich dieselbe aus den Zellkörpern der v. la $\mathrm{V}$ a lett e $\mathrm{S}$ t. Ge or ge'schen Follikelzellen zusammensetzt. Ich muss nach meinen Präparaten an der Deutung der feinen Linien als Zellgrenzen festhalten und kann desshalb Bi o n d durchaus nicht beistimmen, wenn er glaubt, dass das eigenthümliche netzartige Gefüge der Protoplasmamasse einfach der Wirkung bärtender Agentien seine Entstehung verdankt. Dazu verlaufen die hellen Linien doch zu bestimmt und regelmässig und ausserdem gelingt es häufig, mit Hilfe der feinen Contourlinien eine Follikelzelle mit ihrem Kerne vollkommen abzugrenzen. Dass die feinen Liniensysteme dabei namentlich gegen das Kanälchencentrum kernlose Protoplasmafelder abgrenzen, darf uns nicht Wunder nelmen; denn die Follikelzellen, zu deren näherer Betrachtung wir nun iibergehen wollen, stellen relativ grosse, längliche Elemente dar, die namentlich gegen das Centrum des Kanälchens lappige Ausläufer besitzen. Numerisch treten im Hoden der neugeborenen Maus die Follikelzellen sehr stark in

1) a. a. 0 . 
Die postfoetale Histiogenese des Hodens der Maus bis zur Pubertät. 433

den Vordergrund, man sieht ihre Kerne allenthalben, obne dass sie dabei eine besondere Anordnung zeigen, an der Basalmembran sowohl, als auch gegen die Mitte des Kanälchens zu liegen; das Centrum selbst ist allerdings stets frei von Follikelzellkernen, hier werden nur die erwähnten Ausläufer angetroffen. Jedenfalls, das lässt sich sicher sagen, sind im jugendlichen Hoden die Follikelzellen weit zahlreicher vorhanden, wie dies eine Vergleichung der Querschnittsbilder (Fig. 1) mit einem Flächenbilde, das dem Hoden einer erwachsenen Maus entstammt, ohne weiteres deutlich machen dürfte (Fig. 2). Was nun die Kerne der Follikelzellen betrifft, so war ich nicht wenig erfrent, in ilnen dieselben eigenthümlichen Nucleolenbildungen aufzufinden, wie ich sie in einer frïleren $A b$ handlung ${ }^{1)}$ als characteristisch für die Kerne der Benda'schen Fusszelle im functionirenden Hodenepithel beschrieben habe. Während aber hier der Kern stets nur einen relativ grossen Nucleolus in seinem Inneren birgt, sehen wir in den Kernen der jugendlichen Follikelzellen deren 3 bis 4 in entsprechend geringerer Grösse (Fig. 3).

Eingebettet in das Lager dieser Zellen erscheint nun in erheblich geringerer Anzahl die zweite Art von Zellbildungen, die Spermatogonien v. la Valette's St. George's in Form kräftig contourirter, relativ grosser Elemente. In einem Gebiete, das wie die Hodenhistologie gerade bezüglich der Nomenclatur eine ziemliche Complication aufweist, dürfte es sich mit Recht empfehlen, neue Bezeichnungen zu vermeiden und soweit thunlich an den alten Namen festzuhalten; gleichwohl möchte ich es nicht versäumen, darauf hinzuweisen, dass die Spermatogonien im jugendlichen und die im funktionirenden Hoden nicht als absolut identische Bildungen aufzufassen sein dürften. Vor allem unterscheidet sie ihre beträchtlichere Grösse und ausserdem erinnern die jugendlichen Spermatogonien mehr an junge Eizellen. Die rundlichen Zellkörper (Fig, 3) zeigen eine deutl:che Schichtung in eine innere dicht und eine periphere locker genetzte Protoplasmaanlage und auch der Kern lässt die durch ibre Färbbarkeit mit Saffranin so deutlich sich characterisirenden echten Nucleslen der Spermatogonien im erwachsenen Hoden vermissen, er birgt vielmehr in einem ziemlich dichten chromatischen Netzwerk nur einen oder mehrere derbe Chromatinbrocken. Aeusserst zablreiche Mitosen (Fig. 1)

1) a. a. 0 . 
zeigen nun, dass die jugendlichen Spermatogonien in einer regen Vermehrung sich befinden und ist es namentlich das Stadium des Monasters, das sehr häufig zur Beobachtung gelangt.

Dieses, nach den Befunden am Hoden der neugeborenen Maus geschilderte Stadium bleibt nun lange Zeit hindurch bestehen; der Hoden von 9, 12 und 14 Tage alten Mäuschen hat sich zwar als ganzes Organ etwas vergrössert, allein seine histologische Structur zeigt noch ganz dieselben Verhältnisse und auch die Dickendimension der einzelnen Samenkanälchen - im Mittel 0,02 mm - ist die gleiche geblieben wie beim neugeborenen Thiere.

Am 15. oder 16. Lebenstage ändert sich dies aber mit einem Schlage und wir können nun an diesem Tage bei der Maus den ersten Beginn der Pubertätsentwicklung constatiren. Sehen wir nun zu, welehe feineren Vorgänge sich bei diesem Process an den epithelialen Elementen des Samenkanälchens abspielen.

Hatten die Kerntbeilungen der Spermatogonien bis dahin nur stets zur Erzeugung gleichwerthiger Tochterzellen Veranlassung: gegeben, so sehen wir nun, dass durch sie eine Brut von Zellen entsteht, die sich in mehreren Punkten von der Mutterzelle unterscheiden. Diese jungen Zellen (Fig. 4) sind kleiner, sie haben das eiähnliche Aussehen ihrer Mutterzellen verloren und zeichnen sich durch den Besitz mehrfacher, wohlentwickelter echter Nucleolen aus, mit einem Worte, sie stellen Zellen dar, welche den Spermatogonien, wie wir sie in Hoden des erwachsenen Thieres finden, auf das Genaueste gleichen. Die Erzengung solcher Spermatogonien ist dabei eine so rapide, dass wir dieselben in 3 bis 4 fachen concentrischen Schichten iibereinander gelagert finden. Natürlich muss dieser Process der Spermatogonienbildung auch seinen Einfluss äussern auf die zweite Art von Zellen, die Follikelzellen; durch die mächtige Neubildung eigentlicher Samenzellen müssen dieselben auseinander gedrängt werden, so dass ibre Zabl nun hinter der der Spermatogonien zurücksteht, wodurch das numerische Verhältniss zwischen den beiden Zellarten mehr und mehr dem bei dem erwachsenen Thiere entspricht. Auch in Bezug auf die Lage der Follikelzellen innerbalb des Samenkanälchens ist eine Aenderung eingetreten, indem dieselben durch die rasch wuchernden Spermatogonienfamilien aus ihrer Lage im Innern des Samenkanälchens herausgedräugt und allmäblich siimmtlich an die Membrana propria angepresst worden, wo sie 
nun in einer einzigen Schichte ihre Lage haben. Ihre oben erwähnten, gegen das Kanälchencentrum gewandten Fortsätze kommen dabei mehr und mehr zum Schwunde; während man dieselben anfangs noch als mehr oder minder verdünnte Streifen von Protoplasma zwischen benachbarten Spermatogoniengruppen nach dem Centrum zu ziehen sieht, werden sie später von dem kernhaltigen Theile der wandständigen Follikelzelle vollkommen abgelöst und es stellen ihre Reste nun eine Eiweissmasse dar, die gewissermassen immer mehr in sich zusammensintert und so bald zur Bildung eines anfangs buchtigen Lumens im Inneren des ursprünglich soliden Samenkanälchens Veranlassung gibt. Mit diesem Lagewechsel geht auch im Inneren des Kernes der Follikelzelle eine Aenderung Hand in Hand, insoferne als die, wie erwähnt, mehrfach vorhandenen Nucleolen sich zu einem einzigen, grösseren umwandeln und zwar erfolgt dies, wie ieb direct beobachten konnte, durch eine einfache Verschmelzung (Fig. 5).

Nicht alle Kanälchen des Hodenquerschnittes bieten übrigens das ebenbeschriebene Bild; während sich noch viele in dem ursprïnglichen, jugendlichen Stadium befinden, sind andere schon in ihrer Entwicklung vorangeeilt. Recht bäufig kommen Kanälchen vor, in denen sich die Spermatogonienkerne, in 2 bis 3 Schichten gelagert, in die characteristischen enggewundenen Knäuel der sog. growing cells umgewandelt haben (Fig. 6), während stets noch eine Schichte nicht verwandelter Spermatogonien wandständig liegen bleibt und $\mathrm{da}$ ind dort treten Samenkanälchen auf, deren Zellen sich schon in die nächst höbere Zellcategorie, in Spermatocyten, umgebildet haben (Fig. 7). Dass durch diese regen Wachsthumserscheinungen eine Vergrösserung nicht nur des ganzen Hodens, sondern auch der einzelnen Kanälchen bewirkt wurde, muss als selbstverständlich erscheinen; der Querdurchmesser der einzelnen Samenkanälchen beträgt nun im Mittel $0,047 \mathrm{~mm}$.

Ist nun die Entwicklung des Samenkanälebens soweit gediehen, dass die characteristischen lockeren Spirembildungen der Spermatocyten aufiretreten sind, so scheint eine relativ lange Ruhepause eiuzutreten, denn man findet in der Zeit bis zum 21. Lebenstage ausser diesen Spiremstadien keine anderen Theilungsphasen der spermatocyten. Es diirfte vielleicht von allgemeinem histologischen Interesse sein, auf die Möglichkeit einer solch' 
langen Persistenz e in er Kerntheilungsphase hinzuweisen; man hat ja aus dem Umstand, dass im erwachsenen Hoden die Spiremstadien numerisch so sebr über die ubrigen Phasen der Mitose ïberwiegen, schon den Schluss gezogen, dass im Stadium des Spirems eine Ruhepause erfolgen müsse, allein wie lange diese wäbrt, liess sich selbstredend nicht entscheiden. Die Untersuchung des wachsenden Hodens vermag uns in dieser Frage eine directe Antwort zu geben, wir sind zu der Annahme bereclitigt, dass die Spermatocyte bei ihrer Theilung zum mindesten 5 Tage lang in der Prophase des Monospirems verharrt, ehe sie sich anschickt, in die Metakinese und die sich anschliessenden Anaphasen überzugehen. Diese erscheinen erst am 21. Tage und müssen sich dann um so rapider abspielen, denn man sieht an diesem Tage schon da und dort Gruppen neugebildeter Spermatiden. Die Hauptentstehungszeit dieser letzteren liegt von dem genannten Tage bis zum Schlusse der 4. Lebenswoche und unterscheiden sich die neugebildeten Spermatiden in keiner Weise von denen des erwachsenen Thieres; anch sie bergen die beiden Protoplasmaeinschlüsse, die Koptkappenanlage und den Nebenkern, deren Entstehangsmodus mir aber auch hier leider nicht zu beobachten gelang. Die Dicke des Samenkanälchens hat durch diese Ausbildung der Spermatidengenerationen im Mittel 0,078 mm erreicht.

Mit Schluss der 4. Lebenswoche ist also der erste Process, den wir im erwachsenen, functionirenden Hoden als regeneratorischen auffassten, und der mit der Neubildung von Spermatogonien beginnt, mit der Entstehung von Spermatiden endigt, beim jugendlichen Thiere abgeschlossen. In der 5. Lebenswoche setzt nun der zweite Process, der eigentliche Samenbildungsprocess, ein, es bilden sich die Spermatidengruppen in früher beschriebener Weise ${ }^{1}$ ) um, treten mit den Benda'schen Fusszellen in Contact und wir finden in der 6.' Woche schon sämmtliche Entwicklungsphasen der Spermatosomen bis zum fertigen, im Lumen der Samenkanälchen liegenden Spermatozoon.

Mit der 6 . Woche hat also die Maus schon die rollständige Geschlechtsreife erreicht; ob sie freilich in diesem relativ jugendlichen Alter factisch schon das Begattungsgeschäft besorgt, vermag ich nicht zu entscheiden, beobachtet habe ich es selbst nie.

1) a. a. 0. 


\title{
Erklärung der Abbildungen anf Tafel XXVI.
}

Sämmtliche Figuren sind unter Benutzung einer apochrom. Oelimmersionslinse von Zeiss Ap. 1,2. Brenuw. 2,0 und Ocular 4 und 12 mit der Camera lucida entworfen. Vergrösserung 500-1500.

Fig. 1. Querschnitt durch ein Samenkanälchen der neugeborenen Maus 500/1. fz. Follikelzelle. Sp. Spermatogonie.

Fig. 2. Tangentialschnitt durch ein Hodenkanälchen der erwachsenen Maus. $500 / 1$.

Fig. 3. Eine Spermatogonie und 2 Follikelzellen von der neugeborenen Maus bei stärkerer Vergrösserung. 1500/1.

Fig. 4. Neugebildete Spermatogonienschichten. Maus, 16 'Tage alt. 500/1.

Fig. 5. Vereinigung zweier Nucleolen zu einem einzigen grösseren. Maus, 16 Tage alt. 1500/1.

Fig. 6. Unwandlung der Spermatogonien in sog. growing cells. Maus, 16 Tage alt. 500/1.

Fig. 7. Umwandlung der growing cells in Spermatocyten. Maus, 16 Tage alt. 500/1.

Sämmtliche Präparate waren mit Chromosmiumessigsäure gehärtet und einer Doppelfärbung mit Saffiranin-Gentianaviolett unterzogen worden.

\section{Amitotische Kerntheilung im Blasenepithel des Salamanders.}

\author{
Von
}

W. Flemming in Kiel.

Hierzu Tafel XXVII.

Eine vorläufige Mittheilung über das Folgende wurde auf dem 3. Anatomencongress in Berlin (Sitzung rom 10. October 1889, s. im Anatom. Ánzeiger 1889) gegeben.

In einer Harnblase von Salamandra maculosa, die in der gewöhnlichen, für Flächenpräparate früher von mir angegebenen 
Archiv-fimikroskop. Anutomie. Bd.LLTW.

Tof. LTT T.

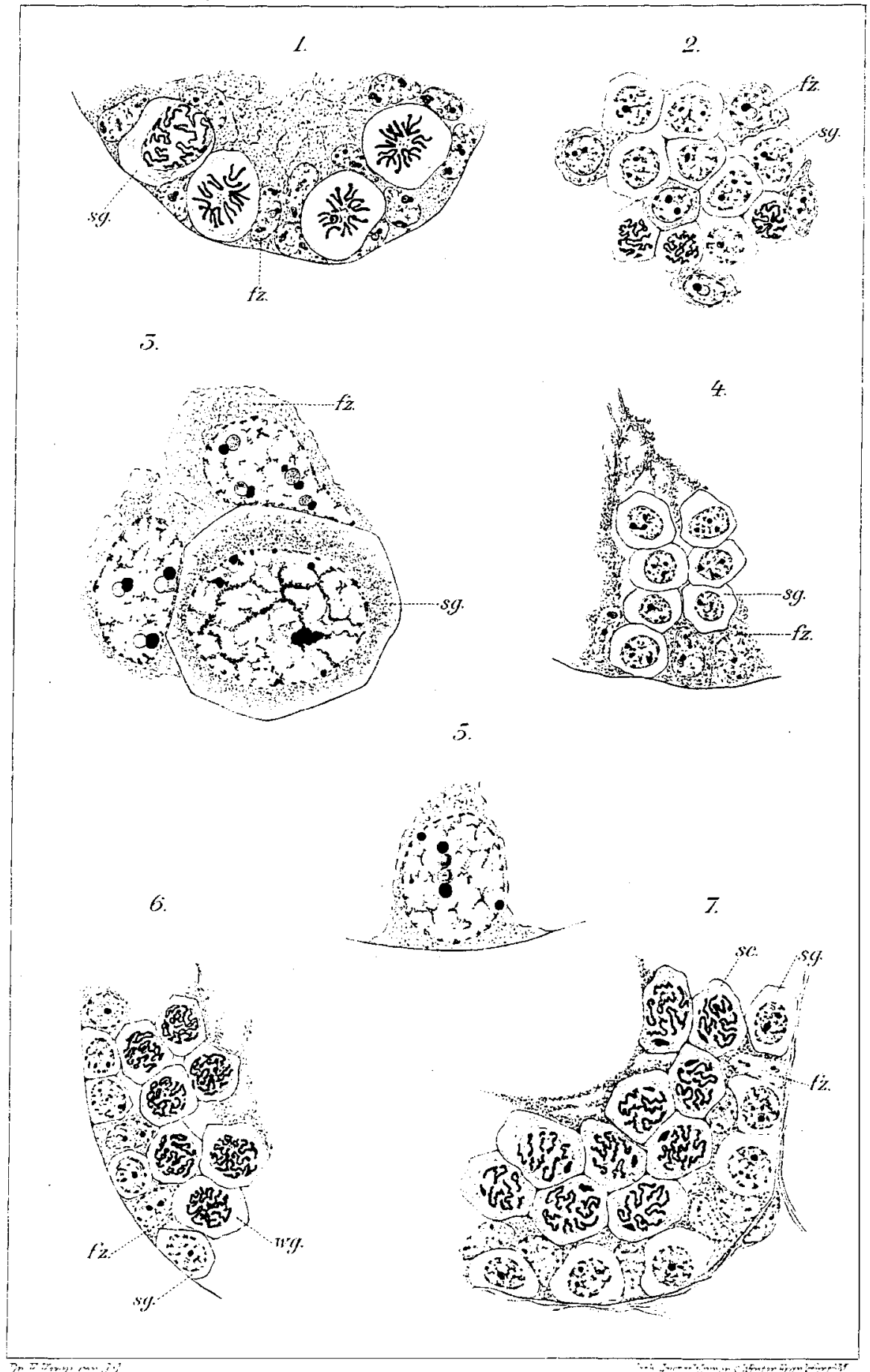

